\title{
MANUALES Y TEXTOS DE ESTUDIOS DE LA UNIVERSIDAD DE CÓRDOBA, ARGENTINA EN EL ULTIMO TERCIO DEL SIGLO XIX
}

\author{
María Cristina Vera de Flach \\ CONICET-Universidad Nacional de Córdoba \\ vera@onenet.com.ar
}

\section{RESUMEN:}

La Ilustración trajo consigo un nuevo instrumento para utilizar en el dictado de las asignaturas universitarias: los libros de texto. A partir de entonces estos comenzaron a circular asiduamente entre docentes y estudiantes. En consecuencia, hubo una proliferación de impresos y una mejora de las bibliotecas universitarias, las que debían ponerse al servicio de la docencia y los usuarios. En el último tercio del siglo XIX y partir de la reforma de Lucero se incentiva la producción de textos de estudio en la Universidad de Córdoba, algunos fueron fruto de traducciones de libros europeos pero, en otros casos, fueron producto del trabajo intelectual de sus profesores. De hecho hubo varios de baja factura pero, otros, suponían una verdadera ruptura con el pasado y una apertura liberal, en tanto modificaban conceptos atrasados y presentaban los resultados de una ciencia que pretendía entrar a la modernidad.

Palabras Claves: Ilustración; manuales de cátedra; docentes

\section{TEXTBOOKS AND STUDY-MANUALS OF THE UNIVERSITY OF CORDOBA, ARGENTINA, IN THE LAST THIRD OF THE XIX CENTURY}

\begin{abstract}
:
The Age of Enlightenment introduced a new resource for use in University assignments: textbooks. From that time onwards, textbooks began to circulate amongst professors and students alike. As a consequence of this, there was a proliferation of printing houses and an improvement in University libraries, which were placed at the service of professors as well as other users. In the last third of the XIX century, following on from Lucero's reforms, the production of textbooks was encouraged within the University of Cordoba itself. Some were the fruits of translations of European books, while others were the intellectual products of the University's own professors. Whilst there were some of rather limited value, others represented a genuine break with the past, opening up a more liberal path; particularly in so far as they modified backward concepts and presented their results within a science intent on entering the modern age.

Keywords: Age of Enlightment; Academic/Departmental Manuals; professors
\end{abstract}

\section{Nuevos proyectos, nuevos tiempos}

Hasta fines de la década de 1860 la Universidad de Córdoba de la República Argentina, poseía una estructura simple, con una matrícula relativamente pequeña monopolizando los estudios la carrera de abogacía. Las aspiraciones de los jóvenes de entonces se limitaban a obtener un título profesional con el que podían tener acceso a diversas funciones administrativas, muchas de las cuales les permitían tener acceso a la elite de poder. 
Hacia la misma época, el Dr. Manuel Lucero, hombre de Justo José de Urquiza y amigo y cofrade de Domingo F. Sarmiento, de ideas desbordantes, intelectual y conocedor de la política de su tiempo, después de la muerte del primero, decidió dar un vuelco a su vida aislándose de la política, por lo que pensó que para poder sobrevivir ejercería como letrado en Córdoba. Con ese fin arribó a esta ciudad en marzo de 1870; sin embargo sus intenciones pronto se desvanecieron cuando, el 1 de enero de 1874, fue designado rector de la Casa de Trejo, nombre con que se conoce a la casa de altos estudios. El camino que se le abría por delante era arduo, en tanto entre sus aspiraciones figuraba el lograr la modernización y renovación de la misma llevando a cabo la primera gran reforma, después de la realizada por el Deán Gregorio Funes a comienzos del siglo XIX1.

Para lograr lo que se proponía debía contar con apoyo oficial y en esto mucho tuvo que ver Sarmiento quien tenía en claro que había llegado el momento en que el país hiciera suyos los progresos científicos de los Estados más adelantados del orbe. Su viaje a Alemania, en 1847, lo había puesto en contacto con la intelectualidad y los científicos de la Universidad de Gottinga a quienes describió "como sabios que vivían lejos del bullicio de las grandes ciudades, sin el aguijón del lujo y enteramente consagrados a las virtudes del claustro". Probablemente recordando esa experiencia, el sanjuanino decidió tomar contacto con Hermann Burmeister, un científico alemán residente en Buenos Aires desde 1861 que tenía a su cargo la dirección del Museo Nacional2. Su autoridad científica era una garantía para solicitarle la opinión sobre lo que debía hacerse para modernizar la Universidad de Córdoba y a través de ella modificar la mentalidad de la población, a la que consideraba influenciada por una tradición religiosa muy fuerte, fruto de la educación jesuítica.

La respuesta le llegó en octubre de 1868, de manos del alemán, cuando en un detallado Informe le sugería organizar en Córdoba un centro educacional con el fin de promover el estudio de nuevas ciencias, expresando:

No existe en la República Argentina un establecimiento que responda a esta elevada tarea. La Universidad de San Carlos en Córdoba está desposeída de catedráticos para todas las ciencias teóricas y exactas; ni las matemáticas, ni la química, ni las otras ramas de las ciencias físicas pueden estudiarse allí; como tampoco es posible en ella el estudio de la historia y de las lenguas antiguas, el griego y el latín, con sus ricas literaturas que han conservado, aun en la oscuridad de la Edad Media, la civilización entre las naciones europeas. Es este un defecto muy grande, que debe ser reparado cuanto antes, y para cooperar a la reforma que requiere, con mis débiles conocimientos me presento a Ud. solicitando la reforma de ese establecimiento colocándolo sobre las bases que estén más en relación con las necesidades modernas y elevándolo a la altura de una verdadera Universidad. 3

El informe de Burmeister y la decisión clara del rector Lucero y el apoyo de la Nación permitió que aquél dos años después fuese designado Comisario extraordinario para dirigir e inspeccionar los cambios en la Universidad de Córdoba. Ante la carencia de una tradición científica en el país, se lo autorizó a contratar siete profesores europeos para que se encargaran de las tareas docentes y de investigación en las nuevas carreras a crearse. A pesar de ser un momento difícil para la Nación, en tanto se afrontaba una guerra contra el Paraguay y una severa epidemia de fiebre amarilla, Sarmiento prestó su apoyo al proyecto en tanto estaba convencido que con él no sólo se daba un paso adelante en la ciencia argentina sino que el mismo serviría para romper con la tradición clerical de la 
ciudad de Córdoba y de su Universidad. Términos que reiteró años después cuando, en un artículo sentenció, que ésta era una de las provincias más atrasadas e ignorantes "resultado de tres siglos de educación jesuítica, franciscana y conventual". Casi de igual manera se expresó su ministro de educación, Nicolás Avellaneda, en 1872, en su memoria al Congreso. Cuando visitó por primera vez a la Universidad en 1870, la encontró reducida a un número menor de cátedras que las que le habían sido atribuidas por la Real Cédula de su erección:

Tenía una cátedra de Filosofía, otra de Matemática y Física y tres de Derecho. La biblioteca con su escaso y antiguo catálogo, no contenía cien volúmenes que sirvieran para auxiliar eficazmente la enseñanza de los profesores $(\ldots) 4$.

Es decir, tanto Sarmiento como B. Mitre, Avellaneda y tantos hombres de entonces tenían en claro que la difusión del conocimiento científico era una cuestión de política de Estado. Obviamente hubo gran resistencia a estos planteos y a los que vendrían y en respuesta se acusó a quienes participaron de dicho proyecto de pertenecer a la masonería, lo que era cierto; al punto que los "hombres de escapulario", como denominaba a los católicos un periódico de la época, llegaron a tildar al rector Manuel Lucero de "Lutero cordobés"5.

La Logia Docente, definida por Emilio J. Corbiere como una organización masónica de elite, contaba entre sus miembros a los antes nombrados6. Si bien no existen registros de su actividad, está claro que la misma tuvo una inserción política y cultural llamativa en Córdoba.

Sus miembros apoyaron el liberalismo económico y al laicismo, participando del Congreso pedagógico de 1882. Pero lo que nos interesa destacar es que algunos de los que colaboraron activamente para poner en marcha el proyecto masónico y laicista se habían iniciado previamente en otras logias provinciales, tal el caso de algunos encumbrados cordobeses que hacia 1867 lo hicieron en la Logia Piedad y Unión $N^{o} 34$. Entre esas figuras destacamos al que sería gobernador de la provincia en 1880 y luego presidente de la Nación, Miguel Juárez Celman, quien apoyará desde esos cargos el proyecto educativo de la Universidad cordobesa.

La decisión con que estos hombres emprendieron los cambios, impulsó la reforma de 1870 en esa casa y una de las razones que coadyuvó para que pudiera realizarse fue porque ese tiempo histórico coincidió con el inicio de los gobiernos liberales. La elite local, imbuida de esas ideas, propugnaba el éxito de la iniciativa privada para las cuestiones económicas, apostando a un mundo desteologizado, evolutivo y progresista, donde el conocimiento científico y tecnológico fuese apropiadamente impartido. Sus representantes, provenientes de distintas corrientes de pensamiento, en el que según señalamos se incluía la masonería, tenían en claro que el camino iletrado y no académico sobre todo en el ejercicio de ciertas profesiones era impensable, peligroso y arcaico.

\section{Arriban los primeros científicos}

Desde febrero de 1870, comenzó a arribar a la provincia mediterránea el primer grupo de científicos contratados en diferentes centros universitarios europeos, en su mayoría procedentes de Alemania, el que estuvo conformado por Paul G. Lorentz, Max Siewert, Wilhem Stelzner, C. Schultz Sellack, Christian August Vogler y Georg Hieronymus. La excepción la constituyó Hendrick Weyenbergh, un zoólogo holandés de nacimiento pero con formación alemana, y un español republicano, Juan Bialet Massé, que se unió años después. 
Todos ellos dejaron su terruño y sus raíces por diversas circunstancias. Algunos lo hicieron por razones personales, otros por la decepción política que vivieron en su propio país como consecuencia de los conflictos de 1848 y, en el caso del español, por el fracaso de la revolución liberal de 1868, pero en el fondo a todos los unían las ansias de lograr en esta región del continente americano nuevos descubrimientos. A la vez los motivaba el deseo de ampliar sus propias investigaciones, lograr su crecimiento intelectual pero simultáneamente los motivaban sus deseos de enseñar y trasmitir sus conocimientos.

Sin conocer el idioma y lo que les esperaba en el mundo americano; desafiando tempestades y los inconvenientes de una larga travesía marina pero llenos de coraje y guiados por una cuota de romanticismo pensaron que ellos iban a poder introducir en el país los estudios de Astronomía, Botánica, Geología, Matemática, Medicina, Paleontología y Zoología.

Eran hombres modestos, que presuponían que iban a ser recibidos con entusiasmo por la sociedad local, sin embargo poco tardaron en decepcionarse y comprobar que ni siquiera el estado nacional cumplía con todo lo que les había prometido: entre otras cosas, porque la fiebre amarilla lo obligó a gastar sumas importantes para hacerle frente y ello retrasó el pago de sus sueldos en varios meses.

De todos modos al llegar varios efectuaron diversos viajes de exploración por el interior del país del mismo modo que lo hicieron varios científicos en Chile, Perú o Brasil. La intención era dar a conocer los resultados de sus investigaciones en trabajos científicos, a la vez que las excursiones les permitirían acumular tesoros invalorables para las diferentes ciencias. Con esos elementos salvados de la destrucción del tiempo y la ignorancia, luego, formaron una base sólida de colecciones con las que - a pesar de los escasos presupuestos - abrieron los Museos y Gabinetes que aun hoy perduran en esta Casa al punto que las generaciones siguientes de universitarios continuarían - sobre la base de esas primeras investigaciones - los estudios sobre el suelo y el hombre americano.

Pero las ideas conservadoras de la sociedad local y de la propia Universidad también coadyuvaron para que muchos sufrieran decepciones, en tanto poco propicia al cambio y a las novedades científicas que ellos trataban de introducir los criticaron a través de la prensa local. En poco tiempo, muchos se vieron envueltos en conflictos. El año 1874 será aciago para los anales de la Historia de la Universidad. Por problemas con Burmeister quien, entre otras cosas, pretendía que los científicos privilegiaran sus tareas docentes por encima de sus investigaciones, Siewert, Weyenbergh y Vogler fueron separados de sus respectivos cargos. Ante esta situación casi todos, en respuesta, presentaron sus renuncias y regresaron a su patria natal.

Más tarde y debido a la presión que ejerciera la opinión pública, en tanto algunos pocos se habían percatado de lo beneficioso que era contar con la presencia de estos científicos, Herman Burmeister renunciaría.

El entonces rector de la Universidad, Manuel Lucero, puso fin al conflicto y decidió separar las actividades de la Facultad de Ciencias Exactas, Físicas y Naturales de las de la Academia de Ciencias. Luego reforzó el plantel de profesores que había quedado acéfalo con la contratación de Adolf Doering que reemplazó a Siewert, Hieronymus a Lorentz. Schulz Sellack fue sustituido por Oskar Doering, quien se hizo cargo de la cátedra de Vogler hasta la llegada de Francisco Latzina. Para sustituir a Stelzner se contrató a Ludwig Brackebusch quien interesó a Artur von Seelstrang para que dictase las cátedras de Topografía I y II7.

Lentamente en los años subsiguientes los jóvenes estudiantes fueron inscribiéndose en las Facultades de Ciencias Físicas y Matemáticas y en la de Medicina. 
Superados los obstáculos iniciales, los profesores que sabían cuál era el rol que debían cumplir para no transformarse en meros trasmisores de conocimientos a través de lecciones magistrales decidieron utilizar nuevas técnicas y estrategias metodológicas para difundir los nuevos conocimientos de las diferentes ciencias a la sociedad en general a través de conferencias, las que, en su mayoría, se dictaron en la Universidad y cuyos textos también se editaron entonces. Ellos estaban convencidos que este tipo de disertaciones que tanto éxito había tenido en Alemania, Estados Unidos e Inglaterra, resultaban útiles para extirpar errores de conceptos en la población.

\section{EI manual o libro de estudios}

A la hora de plantearse nuevos cambios en la Universidad el tema del libro no fue un asunto menor. El proyecto educativo liberal exigía contar con libros de textos para la enseñanza y ante la carencia de éstos en la biblioteca universitaria, que "no correspondía a las pretensiones de una universidad de rango o academia" como se hizo notar en las discusiones del claustro, los docentes recién llegados comenzaron a trabajar arduamente en ese sentido para superar la falencia. Algunos de los textos que aparecieron fueron fruto de traducciones de libros editados y utilizados en universidades europeas. En otros casos, esos textos fueron producto del trabajo intelectual de los profesores titulares de las diversas cátedras. De hecho hubo varios de baja factura pero, otros, suponían una verdadera ruptura con el pasado y una apertura liberal en tanto modificaban conceptos atrasados.

Por razones de tiempo y espacio haré mención a sólo a algunos de ellos dando ejemplos de los utilizados en las distintas Facultades.

\section{Los textos de la Facultad de Derecho}

Si bien es cierto que la Facultad de Derecho era por demás tradicional y que no receptó en esa época entre sus profesores a científicos extranjeros como las restantes Facultades, con excepción del español Dr J. Bialet Massé, hubo docentes que si bien no dejaron producción propia se animaron a cuestionar la enseñanza del derecho tradicional e introdujeron un cambio de orientación en sus cátedras, particularmente en aquellas influenciadas por las profundas transformaciones que experimentaba la estructura social y económica del país en ese período como consecuencia de la influencia del liberalismo político y económico europeo en la opinión ilustrada de la época. No obstante, debemos acotar que algunos autores tardaron en ser aceptados, tal el caso del norteamericano Henry George que estructura la teoría que se conoce por su nombre y que escribió La cuestión de la tierra.

Los inicios de la enseñanza formal de la economía política están ligados a las escuelas de derecho las que se cuentan entre las primeras en las viejas universidades, luego de las cátedras de teología, filosofía y latín. Los primeros intentos de enseñanza formal de la economía política en el Río de la Plata datan de 1822 en la Universidad de Buenos Aires. En la de Córdoba la materia se dicta dentro de la cátedra de Derecho Público - creada por decreto del gobernador de la Provincia de Córdoba, José Antonio Reynafé, el 28 de Febrero de 1834 - con la modalidad de que la cátedra se dictaría en tres años dedicándose el tercero a economía política. La cátedra de Derecho Público contó entre sus primeros catedráticos a los doctores Santiago Derqui, Agustín Pastor de la Vega, Enrique Rodríguez y Ramón Ferreira, no aclarándose cuál o cuáles de ellos dictaría economía política y recomendando que se utilizara el texto que oportunamente fijara el catedrático. La cátedra de Derecho Público y, por consiguiente, la enseñanza de economía 
política perduró hasta el 3 de marzo de 1841, cuando a pedido del claustro universitario fue suprimida por decreto del gobernador delegado Claudio Antonio Arredondo".

El 20 de mayo de 1854 el gobierno de la Confederación nacionalizó la Universidad Mayor de San Carlos de Córdoba y, en 1856, se vuelve a introducir el dictado de Economía Política como cátedra independiente y, por la reforma de 1857, la misma se dicta en tercer año. El 6 de enero de 1870, siendo Presidente de la Nación Domingo Faustino Sarmiento y Ministro de Justicia, Culto e Instrucción Pública Nicolás Avellaneda, por decreto se estableció un nuevo plan de estudios para la mencionada Facultad señalándose los libros de texto que han de emplearse. Economía Política se mantiene en tercer año, adoptándose como texto el Tratado de Courceil Seneuil o el de Garnier en la última edición. En 1887, con el propósito de "uniformar la enseñanza de esta Facultad de Derecho con la de la Capital de la República de conformidad al Estatuto Universitario y la ley de presupuesto", el plan de estudios de la carrera quedó establecido en cinco años, quedando la mencionada cátedra en el quinto año de la carrera. El 14 de marzo de 1889 se la introduce en segundo año.

En esta cátedra los estudiantes cuestionaron el libro de José Garnier para Economía política argumentando que solo existían cuatro ejemplares en Buenos Aires además de estar editado en otro idioma que no conocían. No obstante, debemos reconocer que el autor había puesto todo su empeño para que este Compendio se distinguiera de los demás por las definiciones escogidas, por el buen orden en las materias, por el enlace de las proposiciones aceptadas y de los problemas resueltos, por la claridad y exactitud de las demostraciones y la sobriedad en los hechos y en las cifras. En fin, sostenía que se había esforzado por conservarse en la ortodoxia científica. Sin embargo, "el tratadito elemental, que presentaba tenía únicamente por objeto enseñar a los jóvenes a leer los libros de los maestros y a escuchar con aprovechamiento sus lecciones. No tenía más pretensión que la que indicaba su título; estaba destinado a servir de primera lectura a los que habían oído hablar de la ciencia en bien o en mal, y querían formarse una idea clara de las cuestiones que formaban parte de su patrimonio, a fin de leer luego con fruto las obras para cuya inteligencia se necesitaba tener una preparación, sin la cual es muy expuesto dejarse extraviar". Sabido es que Garnier se basó para editar este manual en los escritos de Quesnay, Turgot, Adam Smith, Malthus, Ricardo, J. B. Say y M. Rossi y de otros libros publicados por otros economistas franceses y extranjeros.

La propuesta estudiantil era cambiarlo por el del célebre economista francés Juan Gustavo Courcelle Seneuil, titulado Tratado de Economía Política, en tanto estaba en castellano y era más completo. Destaquemos que este profesor estuvo contratado por espacio de siete años, entre 1855 y 1863, como asesor del Ministerio de Hacienda del gobierno de Chile además de desempeñarse como docente de dicha Cátedra, desde donde implantó sus principios entendiendo esta asignatura como una ciencia exacta, positiva en sus principios fundamentales8.

La influencia de este francés en el desarrollo de las formas capitalistas será decisiva, imponiendo los principios del laissez-faire laissez-passer, al punto de transformar la estructura mercantil.

En 1883 el del Dr. Juan Bialet Massé se incorporó a la cátedra de Medicina Legal y Toxicología, materia que al poco tiempo fue obligatoria para los estudiantes de quinto año de Derecho y de Medicina. Al abordar la novedosa asignatura éste, que se había formado en España al promediar el siglo XIX, sabía que el médico era un importante colaborador de los juristas, por tal razón decidió que como debía conocer a fondo el origen y naturaleza de las instituciones jurídicas del país, se inscribió para cursar la carrera de Derecho concluyendo sus estudios en solo veinte meses. El 18 de septiembre de 1879 obtuvo el 
grado de Maestro en Filosofía, Bachiller y Licenciado en Derecho Civil y el 25 de octubre de ese mismo año el de Doctor en Derecho Civil.

Compenetrado de la importancia de contar con un manual de estudio para sus estudiantes decidió abordar la redacción de sus Lecciones de Medicina Legal aplicada a la legislación de la República Argentina, obra premiada en el Congreso Médico celebrado en Buenos Aires en 1881 y en un concurso nacional realizado por el Círculo Médico Argentino en 1884. Luego sería declarado de interés por el Consejo Superior.

El manual está dedicado al Dr. Miguel Juárez Celman en "testimonio de gratitud, de amistad y de adhesión" aunque en el prólogo se extiende el agradecimiento al gobernador de la provincia Dr. Gregorio Gavier y a sus ministros Ramón Figueroa y Dermidio A. de Olmos, así como al Decano de la Facultad de Medicina, Dr. Luis Rossi, quienes le prestaron su apoyo. Sin embargo y como ocurre casi siempre con las obras de los docentes universitarios, Bialet Massé debió hacer varias gestiones para que el mismo viese la luz pues él no estaba en condiciones de editarlo lo que relató en una extensa nota donde da cuenta de los costos de una obra de esa naturaleza y de la situación del comercio del libro en la Argentina y las alternativas que debía sortear un profesor universitario a fines del siglo XIX cuando se disponía editar un manual como el suyo.

Cuando encaró su redacción, con excepción de Buenos Aires, en el resto de las provincias argentinas el ejercicio de la medicina no estaba reglamentado, en tanto no habían hecho uso de la facultad conferida por la constitución nacional de dictarse leyes sobre la materia. Por lo tanto se regían de acuerdo a lo que estipulaba la Recopilación Castellana en los títulos 16,17 y 18, libro 5to, puesta en la Recopilación de Indias por las leyes 2 y 7 del título 5to, libro 5to.

Cuando se designó a Bialet Massé para cubrir la novedosa asignatura se lo hizo pensando que la provincia podría contar con nuevos elementos para reglamentar el ejercicio de la medicina y que la justicia contase con profesionales competentes que entendieran en los juicios.

El catedrático se dio cuenta que para cumplir con su tarea debía introducir cambios significativos en la cátedra a su cargo, en tanto se percató que la medicina legal extranjera no era posible de aplicar en la República Argentina, habida cuenta que no se condecía con los Códigos Civil y Penal y las leyes fundamentales argentinas, organizadas jurídicamente a partir de la Constitución de 1853. Por tal razón, sostuvo, era imprescindible redactar un texto de Medicina Legal específico para el país donde se pusiera de relieve esa legislación y se dejasen de lado las soluciones que proponía la Escuela de Francia, principal fuente de la medicina legal hasta entonces.

Y se puso manos a la obra. Tenía conocimiento de la materia en cuestión y ello se demuestra en tanto utilizó como antecedentes la bibliografía más reputada de su época. Recordemos que esta especialidad si bien tenía indicios de existencia en la Edad Antigua y Media tuvo una etapa fecunda en los albores del siglo XVI9, sin embargo es recién a fines del XVIII cuando apareció con base verdaderamente científica en los grandes centros científicos europeos como París, Berlín, Viena, Milán y más tardíamente Madrid. A partir de entonces los médicos legistas llegaron a ser importantes colaboradores de los juristas, transformándose en asesores de la justicia y de los legisladores a la vez que decidían sobre la suerte de enfermos y criminales.

Para definir el concepto de medicina legal, Bialet utilizó la obra del español Pedro Mata y Fontanet, catedrático de medicina legal en la Universidad de Madrid desde 1844, cuyo tratado escrito al año siguiente de obtener su cátedra fue ampliado en sucesivas ediciones y vino a suplantar la obra escrita en 1832 por el abogado madrileño Pedro Miguel de Peiró y el doctor en medicina José Rodrigo, titulada Elementos de medicina y 
cirugía legal. Desde que se hizo cargo de la asignatura Mata se propuso realizar una obra de medicina legal lo más completa posible, tarea que logró a lo largo de su carrera, al punto que L. Comenge y Ferrer reconoció que aquel había sabido "difundir con asombrosa maestría y originalidad esta rama de la ciencia"10.

Pero, si bien Bialet Massé utilizó a Mata, había leído también los autores de la escuela italiana aun cuando ésta sufría un eclipse a mediados del siglo XIX respecto a otras europeas, por eso en su manual citó la obra de Juan Bautista Morgagni, profesor de las universidades de Bolonia y Padua, quien en el siglo XVIII había sentado las bases de la anatomía patológica.

Entre otros autores citados y muchas veces criticados por el autor figuran principalmente los representantes de la escuela francesa, entre los que nombra a E. F. du Salle, Toulmouche, Ambroise Tardieu y otros11. Era consciente que a pesar de contar con estos brillantes expositores dicha escuela padecía de errores graves precisamente por el olvido en que dejaban el estudio de la ley y de su crítica.

En las citas del manual de Bialet Massé figura un solo autor argentino, el Dr. Emilio R. Coni, quien había publicado en Buenos Aires, en 1879, el Código Médico Argentino, texto, que a su criterio, también adolecía de serios errores de derecho proveniente de haber aplicado la jurisprudencia francesa que poseía "leyes tan diferentes a las argentinas", aunque disculpaba a Coni porque era médico.

Las fuentes antes citadas y sus propios estudios le permitieron a Bialet Massé afirmar que el estudio de la medicina legal y la toxicología era imprescindible para los estudiantes de Derecho habida cuenta que los informes médicos dejarían de ser jeroglíficos como lo eran para los que no conocían la materia. Por otra parte, en las cuestiones de responsabilidad podrían adquirir un criterio que no le daba la sicología. Para él la medicina legal era mucho más que el arte de dar dictámenes en juicio, era también la ciencia que podía aconsejar al legislador la sanción y la reforma de leyes fundamentales en los principios de la medicina. En resumen, la materia era necesaria para los abogados que hasta ese momento no estaban preparados para la tarea y para los médicos quienes podrían acudir a ella para realizar los trabajos que el foro les requiriese.

La sistematización es un rasgo destacado de este estudio que ofrecía nuevos temas. El mismo está compuesto de dos voluminosos tomos y se divide en dos bloques que contienen: una parte legal y otra técnica. Con respecto a la primera el autor sostenía que era algo más que la mera transferencia de la ley usada por la escuela francesa, dándole un enfoque naturalista. El derecho, aclaró, se divide en dos ramas generales: la positivista, aquella en la cual la ley está despojada de toda concepción social, política, temporal y la naturalista que es la que considera a la ley como un fruto de un proceso dinámico social en el cual está inmersa y del cual el juez no debe olvidarse a la hora de dictar el fallo judicial.

En su introducción, el autor definió el concepto de medicina legal y sus variaciones según países y legislaciones, señalando la utilidad e importancia de la materia. A continuación hizo referencia al procedimiento médico legal, a la jurisprudencia médica y a los atentados al pudor y las buenas costumbres, manchas de sangre y esperma, para finalizar con los problemas de la reproducción de la especie, el infanticidio y la exposición y sustitución de niños.

La reglamentación del ejercicio de la medicina - tema fundamental en Córdoba desde larga data - fue analizada por Bialet Massé quien, entre otras cosas, señaló la necesidad que el Estado fuese el que controlara las infracciones que cometían a diario curanderos, sangradores, charlatanes, etc.. Sin embargo, él no desconocía lo difícil que era combatir el curanderismo en las localidades alejadas de la capital provincial, en tanto ellas carecían de profesionales en ciencias de la salud con título, razón por la que sugirió que 
transitoriamente podrían habilitarse a aquellos que cursaran una parte de los estudios universitarios con el fin que ejercieran su arte en las regiones donde se los autorizara a ejercer a efectos de satisfacer las demandas de la sociedad. Así se hizo en los años subsiguientes.

La segunda parte del manual contiene todos los adelantos que hasta esa fecha se habían dado a conocer en todos los países y que eran factibles de aplicar en la República Argentina de modo más apropiado que pudiera darse en un curso universitario sobre cuestiones relativas a la identidad, atentados contra la salud y la vida de las personas, las asfixias y la muerte natural, el envenenamiento, las inhumaciones, exhumaciones y autopsias.

En síntesis, la existencia de una obra de estas características era una necesidad sentida tanto para el estudiante de Medicina como para el de Derecho quienes evitaban recurrir a las obras de autores extranjeros, no siempre escritas en castellano. También fue importante para los profesionales que debían tener presente los conocimientos de la materia. La medicina legal siguió en los planes de estudios sin pausa. Los programas positivistas tenían como base la idea que la educación, las buenas leyes y las políticas sociales preventivas podían evitar la necesidad de la represión y mitigar las peores consecuencias de la ignorancia, la pobreza, la desigualdad y la enfermedad12. El estado médico legal demostró ser duradero y el libro de Bialet estuvo vigente hasta 1916 cuando fue reemplazado por el del Dr. Alberto Stucchi.

\section{Los textos de la Facultad de Ciencias Exactas, Físicas y Naturales}

En la Facultad de Ciencias Exactas, Físicas y Naturales los primeros docentes de la casa se caracterizaron por traducir importantes obras que habían tenido éxito entre los estudiantes europeos con el fin de ponerlas al alcance de los jóvenes cordobeses, como así también por redactar las suyas propias, algunas de gran envergadura, tal por ejemplo los escritos de los naturalistas que se ocuparon de la flora argentina y enseñaron las ciencias naturales contándose entre ellos la producción de Pablo G. Lorentz, Jorge Hieronymus, Federico Kurtz y Carlos Berg. En las páginas de muchos de esos escritos se ve la audacia de estos profesores para construir modelos teóricos con el fin de enseñar en la Córdoba finisecular. La similitud de sus carreras académicas e investigativas es un punto en común y ello se advierte en la bibliografía que citan.

Arturo Seelstrang contribuyó permanentemente con su labor a la historia de las ciencias matemáticas en Argentina pues, además de su propia obra, tradujo del alemán, en 1882, Tratado de Trigonometría rectílinea y esférica, de Luis Kambly, de gran éxito en Europa después que los matemáticos alemanes lo habían impuesto en la mayoría de sus universidades al punto que para entonces había alcanzado su 11 edición a raíz de su mérito didáctico13. Este texto era bastante completo teniendo en cuenta que después de los estudios preliminares contenía un detallado estudio de las funciones trigonométricas de los ángulos y la resolución de los triángulos y polígonos regulares, con un apéndice que resumía las fórmulas geométricas más importantes.

Además, en 1883, Seelstrang conjuntamente con Bachmann hizo lo propio con el Tratado completo de análisis algebraico, de H. B. Lübsen, impresión autorizada por el Consejo Superior ostó \$ 800.14 Este libro, editado por la imprenta Coni en Buenos Aires, tuvo varias ediciones en pocos años. Contenía la Introducción al cálculo infinitesimal, cálculo diferencial e integral, lo que posibilitó a los alumnos argentinos el conocimiento matemático de las teorías de los números de Carl Friedrich Gauss y los progresos significativos del autor en Geometría, en las aplicaciones de la Matemática a la geodesia, 
en la teoría newtoniana de la atracción y en el electromagnetismo y en las instrucciones geométricas de Drobish15.

Federico Guillermo Carlos Berg nació en la ciudad de Curlandia rusa el 2 de abril de 1843. En 1871 estuvo en condiciones de ocupar la cátedra de Historia Natural y la de Química en el Politécnico Báltico de esa ciudad, previo examen para obtener el título de profesor, cosa que logró con señalado éxito. Cuando Burmeister se puso en contacto con varios científicos europeos para que le señalaran algunos naturalistas jóvenes para confiarle un cargo en el Museo de Historia Natural de Buenos Aires, el candidato más interesante fue Berg, quien llegó a esa ciudad en 1873 para trabajar como ayudante del sabio germano, aunque también se desempeñó como profesor del Colegio Nacional de Buenos Aires16.

Su obra fue fecunda pero de sus textos destacamos su Tratado Elemental de Zoología y Botánica para uso del alumnado de la cátedra de Historia Natural, utilizado primero en la Universidad de Córdoba y luego en la Facultad de Ingeniería de Buenos Aires donde tuvo a su cargo la misma asignatura. Un volumen con gruesas tapas forradas en tela color carmín ornada con motivos florales en relieve y lomo con letras doradas, fue impreso en Buenos Aires por Ángel Estrada y Cía, en el 466 de la calle Bolívar.

Henry Weyenbergh, de quien nos ocuparemos a continuación, tradujo en forma abreviada e introduciendo algunas modificaciones el libro, escrito en holandés, del célebre profesor de la Universidad de Utrech, P. Harting, titulado Zoología sistemática. Los tres tomos se editaron en Córdoba en la imprenta Rivas en 1877, 78 y 1880 y hacían mención a vol. I Vertebrados; vol. II Invertebrados y el Vol. III Figuras.

Fue además fundador del Periódico Zoológico, órgano de la Sociedad Entomológica Argentina, cuyo primer número vio la luz en Buenos Aires en ese tumultuoso año 1874 de la historia de la Universidad de Córdoba. En ese periódico que salió por entregas irregulares y se editó en Córdoba y Buenos Aires, aparecieron numerosos artículos del mismo Weyenbergh y de otros científicos alemanes, entre los que se cuentan Francisco Latzina y P. P. C. F. Snellen.

Su tiraje era limitado, 100 ejemplares, por esa razón lamentablemente la redacción no podía ofrecer separatas a sus autores, de allí que algunos de los temas que aparecieron sólo es posible rastrearlos en dicha revista. En otros casos hubo autores que decidieron reeditarlos en otras revistas de mayor difusión, como el Boletín de la Academia Nacional de Ciencias de Córdoba. En el caso de Weyenbergh inquirir su producción total se hace aún más difícil, en tanto publicó muchísimos trabajos en revistas científicas de Europa.

Los dos primeros botánicos que arribaron a Córdoba fueron G. Lorentz y Jorge [Georg] Hieronymus, [1846-1921]17. Cuando este último se encontraba en Halle redactando su doctorado en medicina recibió la invitación para venir a Argentina para desempeñarse como ayudante de Lorentz. No dudó un instante y aceptó la propuesta arribando a Córdoba, con escasos 26 años, en septiembre de 1872.

Dos meses después estaba en viaje junto a Lorentz por el norte argentino llegando hasta Bolivia. Fruto de esa expedición, que duró dieciséis meses, publicó, en 1874, en el Boletín de la Academia de Ciencias sus Observaciones sobre la vegetación de la Provincia de Tucumán, reeditadas en 1945 en esa provincia. No fue éste su único viaje sino que siguió recorriendo activamente la Provincia de Córdoba, particularmente sus serranías. Constantemente con Lorentz estuvieron juntando muestras que fueron enviadas al botánico Augusto Enrique Rodolfo Grisebach, considerado como el fundador de la geografía botánica que estaba en Göttingen, para su clasificación, las que constituyeron la base del texto titulado Symbolae ad floram argentinam aparecido en 1879. En escasos nueve años publicó, entre otras cosas, su Monografía de Lilaea subulata, memoria dedicada al 
presidente de la Nación. En el proemio observó la dificultad de escribir con soltura en castellano sobre estos temas, en tanto carecía del vocabulario técnico de la ciencia moderna, sin embargo señaló que se animaba a hacerlo con la esperanza que la publicación contribuiría a fomentar entre los jóvenes ese tipo de estudios:

(...) a la juventud de este país como a la de España y demás naciones en que se habla la lengua castellana, a dedicarse a estudios microscópicos de la clase de éstos y, podrá quizás servir como muestra; modelo y guía en estudios semejantes que quisiesen emprender unos que otros estudiantes de botánica"18.

Debemos, además, a este científico un completo Catálogo de plantas medicinales indígenas por orden de familias, donde llegó a localizar más de 1.200 plantas. También esta obra fue realizada en homenaje a Julio A. Roca como protector de la Academia Nacional de Ciencias de Córdoba. Varios otros artículos de su autoría merecerían citarse, incluido uno de difusión didáctica dedicada a sus alumnos, pero extenderíamos este trabajo más allá de lo aceptado.

Desde la cátedra desarrolló una intensa actividad docente incluso efectuando traducciones del alemán de un Curso de Botánica General para que pudieran utilizar sus discípulos. Hieronymus fue designado miembro de la Academia Nacional de Ciencias donde llegaría a publicar en su Boletín varios artículos que enriquecieron los estudios botánicos de la Argentina.

Antes de partir a Alemania, en septiembre de 1878, decidió donar a la Biblioteca Mayor de la Universidad su propia biblioteca que contaba con un total de 73 obras siendo ésta la primera donación importante recibida por esa institución hace ya más de un siglo. Todo el fondo documental lleva en la contratapa u hoja de guarda lo siguiente: "Regalado a la Biblioteca de San Carlos por Jorge Hieronymus. Septiembre de 1878".

Entre esos libros había una Biblia, 2 obras de historia natural, 1 de astronomía, 2 de química, 3 de geología, 42 de botánica, 4 de zoología, 4 de medicina, 1 de agricultura, 6 de literatura clásica y 1 de historia de las cruzadas. La mayor parte en idioma alemán y de autores alemanes, aunque hay otras en latín y griego, editadas en el siglo XIX o en el anterior, lo que nos hace suponer que ésta debió ser la biblioteca personal que trajo a su ingreso al país. No hay en ella ningún trabajo suyo ni de antes ni a posteriori de su asentamiento en Córdoba, como así tampoco de sus colegas de entonces en la Universidad. Esas obras, tal vez, lo acompañaron en su regreso definitivo a Alemania.

En 1892 ingresó a trabajar como curador en el Museo Botánico de Berlín y en ese lugar completó y estudió las colecciones de dicho Museo. Desde 1893 fue editor de una publicación especializada, la revista Hedwigia, donde aparecieron varias contribuciones sobre las plantas sudamericanas y argentinas. Lamentablemente algunas de sus colecciones argentinas no estudiadas se perdieron durante la Segunda Guerra Mundial. En 1920 sufrió un ataque de apoplejía falleciendo en Berlín el 18 de enero de 1921.

La similitud de la carrera académica e investigativa de Federico [Fritz] Kurtz [1854-1920] es común a los casos antes citados. Oriundo de Berlín, nació el 6 de marzo de 1854 quedando huérfano de madre al poco tiempo. En marzo de 1861, ingresó en la Escuela Real donde cursó los estudios primarios y secundarios, manifestando tempranamente su afición a aprender Ciencias Naturales. En ese lapso tuvo algunos problemas con sus compañeros, como consecuencia de su inclinación a gastar bromas, hecho que, con fina ironía, siguió practicando dentro de los claustros universitarios cordobeses. 
Hacia 1872 ingresó a la Universidad de Berlín teniendo como profesores a hombres notables de su tiempo, entre otros a P. Ascherson, A. Bastian, E. Berich, R. Hartmann, y como su maestro en Botánica a Alejandro Braun. Antes de doctorarse en Filosofía, hecho que se produjo en 1879, trabajó como ayudante de Mineralogía en el Museo y en el Jardín Botánico de esa ciudad.

Posteriormente recorrió el norte de Alemania y otros países europeos llegando a visitar el British Museum. Mientras estaba trabajando en el Museo de Berlín se recibió una carta de Hieronymus donde solicitaba un catedrático de Botánica para la Universidad de Córdoba. Poco tiempo después, en 1884, falleció su padre y luego su madrastra y eso lo decide a embarcarse rumbo a la Argentina donde habría de terminar sus días.

El Dr. Pablo Ascherson fue el encargado de comunicar el 15 de octubre de 1883 al Ministro de Instrucción Pública de la Nación que Kurtz había firmado contrato agradeciendo la oportunidad que se le brindaba y esperando corresponder con su trabajo la confianza brindada cuando emprendiera viaje a esta ciudad19.

Como otros, desconocía el idioma, pero ello no fue un impedimento para cruzar el océano, y poco después formar parte de una expedición científica al Chaco comandada por Eduardo Holmberg y en la que intervinieron, además, Florentino Ameghino, Carlos Galander y Federico Schulz. A partir de allí se inició entre todos ellos una entrañable amistad. En cambio, el hecho de no dominar el castellano le impidió, inmediatamente de su arribo, hacerse cargo de las funciones docentes, lo que ocasionó quejas de parte de su paisano Seelstrang20.

Pronto estuvo Kurtz en condiciones de realizar profundas investigaciones. Sus expediciones no se detuvieron y a lomo de mula, como lo hicieran sus compañeros, recorrería distintas regiones del país con el objeto de reunir el Herbarium Argentinum, el que llegó a contar conde 16.299 ejemplares, con duplicados y preparados en forma ejemplar. Un tiempo largo le llevó conseguir un discípulo que se interesase en continuar con esa tarea, sin embargo logró su meta cuando apareció Alberto Castellanos, a quien entusiasmó en la Botánica aunque era tarde para formarlo personalmente. Eso le hizo decir al momento de su jubilación que no había podido conseguir un sucesor para su cátedra. Hasta su retiro, en 1915, contribuyó permanentemente a enriquecer los conocimientos de la Botánica argentina y sus análisis permitieron demostrar la vinculación de la flora americana con la de la India, Australia y África del Sur, lo que le posibilitó dominar los estudios paleo-botánicos de Argentina. La sistematización de sus investigaciones es un rasgo destacado de este científico que ofreció además varios textos donde hizo sugerencias y planteó hipótesis.

Para entonces estaba convencido que sólo había en el país dos botánicos: Spegazzini y él. Lamentablemente Kurtz no pudo regresar a su patria natal, ni siquiera de paseo, en virtud de estar siempre necesitado de dinero. Los últimos años de su vida no fueron muy productivos, sin embargo enfermo y pobre dispuso con sus últimas fuerzas que su herbario y su biblioteca no se disgregaran. Entonces sentenció: Kurtzi Herbarium et Bibliotheca non disgregantur. Falleció en Córdoba en 1920.

\section{Los primeros textos de la Facultad de Medicina}

En los primeros años de vida de dicha casa de estudios, los avances de la medicina a nivel mundial se conocían en Córdoba a través de unos contados libros que llegaban a la biblioteca universitaria los que, obviamente, estaban en otros idiomas, apreciándose todo a través de las láminas y esquemas que los adornaban.

Finalmente dedicaré unas palabras a uno de los gestores de la existencia de la Facultad de Medicina, el Dr. E [Hendrich] Weyenbergh, (1842-1885), un hombre 
comprometido con su profesión y con Córdoba en particular21. A él le debemos los primeros textos que utilizaron los estudiantes de medicina y las de la escuela de Parteras. Desde temprano se preocupó por poner en conocimiento de los estudiantes cordobeses la existencia de las principales obras de histología del siglo XIX. Basándose en ellas escribió dos manuales editados en esta ciudad. El primero, vio la luz en 1873 y fue editado por la Imprenta Yofre, se titulaba Corporis Humani, Anatomiae Descriptivae, vulgarmente era un tratado de anatomía descriptiva. Fue escrito en latín para uso de sus discípulos. Y, el segundo, editado por la Imprenta de El Interior fue escrito en 1879; era un compendio titulado Principios Histológicos22. Como lo manifiesta en el prólogo de la primera edición las dos primeras partes eran una traducción modificada del libro Grundzuge der Histologie, Einleitung in das Studium desselbern, del profesor Dr. H. Frey en particular, que había sido muy bien recibido en las universidades europeas. Pero Weyenbergh conocía además las principales obras de histología editadas en Europa como las de Rudolph Albert von Kölliker, Franz Leydig, Maestre de San Juan, V. Cornil y Louis Antonine Ranvier Manuel d'histologie pathologique, publicado en Paris, G. Baillière en 1869, aunque a su criterio- algunos de éstos tenían inconvenientes por ser anticuados, confusos o exclusivamente patológicos. Escribió además dos artículos de vanguardia titulados Útero infantil e himen perforado en una mujer casada o Extracción de una cabeza fetal amputada en el útero.

Cuando se inauguró la Cátedra de Obstetricia, que cursaban los estudiantes de sexto año de medicina y las mujeres de la Escuela de Parteras, el Dr. Luis Rossi, un italiano que estuvo al frente del decanato de la Facultad de Medicina en la década del ochenta, tuvo la audacia de redactar un Manual de obstetricia, ginecología menor para las comadronas que sabemos por distintas fuentes fue utilizado en esa etapa inicial pero que no podemos comentar pues lamentablemente no pudimos encontrarlo ni en Argentina ni en Italia.

Al inaugurarse la biblioteca de la Facultad de Medicina comenzaron a llegar los Anales de Ginecología, Revistas especializadas en enfermedades de la infancia, la Semana Médica y otros boletines los que fueron apreciados por profesores y estudiantes 23 .

\section{A modo de Conclusión}

Desde comienzos del siglo XIX, con la aparición del historicismo y del positivismo, el empleo del texto en las cátedras universitarias se incentiva así como la lectura de revistas y actas de congresos especializados.

Los profesores que en Córdoba abordaron la escritura de sus propias investigaciones con el fin de ponerlas al alcance de sus estudiantes, pensaban que el libro podía ser uno de los aldabonazos para concretar la reforma que algunos círculos universitarios reclamaban tímidamente a fines del siglo XIX. En efecto, para entonces eran muchos los estudiantes que manifestaban públicamente que las largas exposiciones de los profesores iban perdiendo su razón de ser en las clases diarias por lo que creían que el uso de un manual de textos y la presencia de revistas especializadas que comenzaron a importarse podían eliminarlas totalmente a mediano plazo habida cuenta que la superioridad didáctica de estos materiales era insuperable. Es que un manual bien hecho, que contuviera conceptos claros y precisos no necesitaba otro complemento, mientras que una clase magistral requería de la lectura para ser aprovechada.

Para poner en práctica este cambio era preciso en primer término que los docentes realizaran trabajos de investigación, los que luego podían dar a conocer en sus propios textos los que, por lo general, no fueron editados por la propia universidad. Es justamente en la preparación de los tratados donde se pueden presentar los resultados de una ciencia madura y completa. En la Facultad de Ciencias Naturales y en la de Medicina ello fue 
posible porque los profesores estaban en condiciones de abordar esa tarea, mientras que en la de Derecho costó más pues había que modificar la mentalidad de los docentes pues ello exigía que éste fuese además investigador, sólo así podía exponer sus conclusiones en un manual. No obstante contamos con varios ejemplos que podrían mencionarse.

La redacción de monografías más pequeñas, algunas expuestas en las conferencias que se dictaron los miércoles en la Biblioteca Mayor de la Universidad, implicaba la existencia de un conjunto de investigaciones serias y bien preparadas sobre temas que eran desconocidos o interesaban a la población en general, las que fueron publicadas en periódicos locales o en pequeños folletos, hoy sumamente útiles para conocer el estado de la ciencia al finalizar el siglo XIX.

A pesar de la importancia de los textos mencionados debemos hacer notar que éstos no fueron definitivos pues cada generación tiene el derecho de hacer su propio aporte y reflexión.

\section{FUENTES Y BIBLIOGRAFIA}

ARCHIVO FACULTAD DE CIENCIAS EXACTAS FISICAS Y NATURALES, [A.F.C.E.F.y N.], Carpeta 34.

Universidad de Córdoba. Documentos

Correspondencia

\section{ARCHIVO GENERAL DE LA UNIVERSIDAD DE CORDOBA[A.G.U.C.]}

Papeles interesantes, B 25

Libro de Actas del Consejo de la Facultad de Ciencias Físico-Matemáticas

Notas diversas, 1874.

Libro 17, 1872-1873 fs. 23

BERMANN Gregorio. La obra educacional del rector Manuel Lucero y su época. Córdoba: Biffignandi, 1922.

BURMEISTER, Hermann. Memorandum al señor presidente de la República antes de tomar posesión de su puesto. Boletín de la Academia de Ciencias de Córdoba, Córdoba, Tomo 1, 1874.

BURMEISTER, Hermann. Viaje por los Estados del Plata con referencia especial a la constitución física y al estado de la cultura de la República Argentina realizada en $1857,1858,1859$ y 1860. v. 1.

CASTELLANO, Alberto. Recuerdos de un viejo solitario. Fritz Kurtz. Buenos Aires: Imprenta de Felipe Garfunkel, 1921.

CORBIERE, Emilio J. La masonería. Política y sociedades secretas en la Argentina. Buenos Aires: Editorial Sudamericana, 1998.

COMENGE Y FERRER L. La medicina del siglo XIX. Apuntes para la historia de la cultura médica en España. Barcelona: Espasa, 1914.

GARCIA CASTELLANOS, Telasco. Sarmiento y su influencia en Córdoba. Córdoba: Academia Nacional de Ciencias, 1988.

Revista HISTEDBR On-line, Campinas, número especial, p. 111-127, mai.2010 - ISSN: 1676-2584 124 
GARZON MACEDA, Félix. Historia de la Facultad de Ciencias Médicas. Córdoba: Imprenta de la Universidad, 1927. 3 vol.

GARZON MACEDA, Félix. La medicina en Córdoba. Apuntes para su historia. Buenos Aires: Talleres Gráficos Rodríguez Giles, 1916. 3 tomos.

SARMIENTO, Domingo Faustino. Obras Completas. Buenos Aires: Imprenta y Litografía Mariano Moreno; Belín Sarmiento editor, 1899. Tomo 48.

SALVATORE, Ricardo. Sobre el surgimiento del estado médico legal en la Argentina. Revista Estudios, Santa Fe, n. 20, 2001.

SECKT, Hans, Friedrich Kurtz. Ein Nachruf Zeitsch Deusch Wissensch. Buenos Aires: 1920.

UDAONDO, E. Diccionario Biográfico Argentino. Buenos Aires: 1938.

VERA DE FLACHS, María Cristina. La Ciencia Joven. Prosopografía y producción científica de los académicos alemanes de la Universidad de Córdoba. 1870-1900. Córdoba: Junta Provincial de Historia de Córdoba, 2002.

VERA DE FLACHS, María Cristina, Científicos alemanes en la Universidad de Córdoba. 1870-1900. In: Jornadas de Historia del Pensamiento Científico Argentino, 5, Buenos Aires, 1993. Actas... Buenos Aires: F.E.P.A.I, 1993.

VERA DE FLACHS, María Cristina. La Ciencia y los científicos alemanes en la Córdoba del XIX. Investigaciones y Ensayos, Academia Nacional de la Historia, Buenos Aires, n. 44, 1994.

VERA DE FLACHS, María Cristina. Científicos alemanes en la Universidad de Córdoba al filo de los dos siglos. Revista Puente, Asociación Ex Becarios de la República Federal de Alemania, Córdoba, v. 1, n. 1, 1996.

VERA DE FLACHS, María Cristina. De Comadronas a Obstetras. La institucionalización del saber. Córdoba: CONICET, 2010.

VERA DE FLACHS, María Cristina. Un español republicano en Argentina: Juan Bialet Massé. Sus textos de anatomía y manual de Medicina Legal. In: Manuales y Textos de enseñanza en la universidad liberal. Madrid: Universidad Carlos III, 2004.

VERA DE FLACHS, María Cristina. Gregorio Funes. Un criollo ilustrado y la reforma del plan de estudios de la Universidad de Córdoba. In: Criollismo Científico. Madrid: Editorial Doce Calles, 2000. 
Notas:

${ }^{1}$ Para quien se interese por el tema cfr. VERA DE FLACHS, María Cristina, Gregorio Funes. Un criollo ilustrado y la reforma del plan de estudios de la Universidad de Córdoba en Criollismo Científico. Madrid: Editorial Doce Calles, 2000. Editores Diana Soto Arango, Miguel Angel Puig Samper y Ma. Dolores González.

2 Burmeister nació el 15 de enero de 1807 en Stralsund. Cursó sus estudios en el gimnasio de su ciudad natal y luego pasó a la Universidad de Greifwold y a Halle donde estudió medicina a la vez que se dedicó a realizar estudios de Historia natural, doctorándose en 1829. Se estableció en Berlín donde se desempeñó como catedrático de dicha cátedra en uno de los gimnasios existentes en esa ciudad. Volvió a Halle en 1837 y en 1842 fue designado profesor de la cátedra de Zoología de la mencionada Universidad. En 1848 tomó parte de las agitaciones políticas de su país, siendo designado miembro de la asamblea nacional alemana y de la Cámara prusiana. En 1850 desilusionado de la política abandonó Alemania para recorrer Brasil, visitando particularmente Río de Janeiro y Minas Gerais. Regresó a Europa y en 1856 viajó a Montevideo y de allí pasó a la República Argentina realizando luego un largo periplo que lo llevó hasta Panamá y Cuba antes de retornar por última vez a su país, para regresar a Buenos Aires donde se radicó definitivamente. VERA DE FLACHS, María Cristina. La Ciencia Joven. Prosopografía y producción científica de los académicos alemanes de la Universidad de Córdoba. 1870-1900. Córdoba: Junta Provincial de Historia de Córdoba, 2002.

${ }^{3}$ BURMEISTER, Hermann. Memorandum al señor presidente de la República antes de tomar posesión de su puesto. Boletín de la Academia de Ciencias de Córdoba, Córdoba, 1874, Tomo 1.

${ }^{4}$ A similares reflexiones había llegado Hermann Burmeister cuando recorrió la provincia de Córdoba, viaje que retrató en su libro titulado Viaje por los Estados del Plata, cuando señaló que la Universidad contaba con un rector y seis profesores ordinarios para Teología, Matemáticas, Derecho canónico, Filosofía y Gramática" y que..." no había cátedras de Historia y de Física, La Universidad solo enseña estudios lucrativos, toda ocupación teórica, puramente científica ha sido eliminada. Así se comprende que seis profesores basten". Cfr. Viaje por los Estados del Plata con referencia especial a la constitución física y al estado de la cultura de la República Argentina realizada en 1857, 1858, 1859 y 1860, vol. I, página 38. Hay edición en alemán publicada por Druck un Verlag con H.W. Schmidt, Halle.

BERMANN, Gregorio. La obra educacional del rector Manuel Lucero y su época. Córdoba: Biffignandi, 1922.

6 CORBIERE, Emilio J. La masonería. Política y sociedades secretas en la Argentina, Buenos Aires: Editorial Sudamericana, 1998. p. 248 y ss.

${ }^{7}$ Para ampliar estos temas remitimos a los trabajos de la autora citados en la bibliografía anexa.

${ }^{8}$ AGUC, Libro 17, 1872-1873 fs. 23.

${ }^{9}$ En 1532 se publicó la Constitutio Criminalis Carolina en la dieta de Ratisbona y la primera obra de Medicina Legal, aunque breve, fue escrita en 1575 por Ambrosio Paré y se tituló Des Rapports et des mohines d'embauer les corps morts.

${ }^{10}$ COMENGE Y FERRER, L. La medicina del siglo XIX. Apuntes para la historia de la cultura médica en España. Barcelona: Espasa, 1914. p. 471.

${ }^{11}$ Du Salle (1796-1873) estudió medicina y lenguas orientales en París. Publicó varias obras, algunas de importancia como Histoire genérale de la médicine légale y otras de menor factura. A. Tardieu también escribió varias que se encontraban en la Biblioteca de la Universidad de Córdoba, entre ellas, Etude mèdico legale sus la folie, Paris, Braillière, 2da edición, 1880.

${ }^{12}$ VERA DE FLACHS, María Cristina. Un español republicano en Argentina: Juan Bialet Massé. Sus textos de anatomía y manual de Medicina Legal en Manuales y Textos de enseñanza en la universidad liberal. Universidad Carlos III, Madrid, 2004.; SALVATORE, Ricardo. Sobre el surgimiento del estado médico legal en la Argentina, Estudios, Santa Fe, n. 20, 2001. p. 98 y ss.

${ }^{13}$ Seelstrang nació en 1808 en Prusia Oriental. En la Universidad de Córdoba tuvo a su cargo varias materias además de desempeñarse como Decano de la Facultad de Ciencias Físico-Matemáticas en los años 1880. Su obra fundamental es el mapa topográfico de la República que realizó por encargo del Instituto Geográfico Argentino. Falleció en esta ciudad el 21 de setiembre de 1896.

14 ARCHIVO FACULTAD DE CIENCIAS EXACTAS FISICAS Y NATURALES. [A.F.C.E.F.y N.] Carpeta 34. folio 253.

${ }^{15}$ Las contribuciones de Gauss a las matemáticas van desde la más pura teoría de números hasta los problemas prácticos de astronomía, magnetismo y topografía. Realizó grandes aportaciones en todas las ramas de las matemáticas en las que trabajó. Llegó a publicar alrededor de 155 títulos, sin embargo su producción fue mayor pues se caracterizó por no presentar los trabajos que no creyera haber pulido hasta la perfección.

Revista HISTEDBR On-line, Campinas, número especial, p. 111-127, mai.2010 - ISSN: 1676-2584 126 
${ }^{16}$ Entre los discípulos argentinos de Berg, sobresalieron con rasgos propios, Eduardo Ladislao Holmberg, Miguel Ignacio Lillo, Cristóbal M. Hicken y Ángel Gallardo.

${ }^{17}$ Jorge Hieronymus nació el 15 de febrero de 1846 en un pueblo de Silesia, hoy territorio polaco, en un hogar de una familia de terratenientes. A una corta edad perdió a su padre, lo que incidió para que su madre junto a sus tres hijos se trasladara a Görlitz, desprendiéndose previamente de su propiedad. En el Gymnasium de ese poblado se recibió de bachiller y a partir de allí decidió continuar sus estudios en grandes centros europeos. María Cristina VERA DE FLACHS, La Ciencia Joven. Prosopografía y producción científica de los académicos alemanes de la Universidad de Córdoba. 1870-1900. Córdoba: Junta Provincial de Historia de Córdoba, 2002. A.G.U.C., Notas diversas, 1874.

18 HIERONYMUS, G. Monografía de Lilaea subulata. Actas de la Academia Nacional de Ciencias de Córdoba. Buenos Aires: Imprenta Coni, 1882. Con dibujos del autor y litografía de Duval de Berlín.

19 El Interior, diario de Córdoba, 20 de octubre de 1883.

${ }^{20}$ SECKT, Hans. Friedrich Kurtz. Ein Nachruf Zeitsch Deusch Wissensch. Buenos Aires: 1920. También se ha ocupado de este científico su amigo CASTELLANO, Alberto. Recuerdos de un viejo solitario. Fritz Kurtz. Buenos Aires: Imprenta de Felipe Garfunkel, 1921.

${ }^{21}$ Weyenbergh nació en Harlem, Holanda, donde se recibió de médico aunque se especializó en zoología en las Universidades de Utrech y Göttingen. Llegó a Córdoba en 1872. Cuando la facultad de Medicina abre sus puertas fue designado decano, formulando su plan de estudios. La universidad reconoció los esfuerzos realizados y lo premió con el título de Doctor Honoris Causa en virtud del alto nivel científico que se encontraba esta Facultad y a la valiosa cooperación que él supo dispensarle.

${ }^{22}$ La primera edición de los Principios Histológicos fue publicada como folletín en los diarios El Progreso y El Interior.

${ }^{23}$ VERA DE FLACHS, María Cristina. De Comadronas a Obstetras. La institucionalización del saber. Córdoba: CONICET, 2010; GARZON MACEDA, Félix. La medicina en Córdoba. Apuntes para su historia. Buenos Aires: Talleres Gráficos Rodríguez Giles, 1916. 3 tomos.

Artigo recebido em: 25/04/2010

Aprovado para publicação em: 26/05/2010 